\title{
Smart Home System Based on Wireless Sensor
}

\author{
Yan $\mathrm{Wu}^{1, \mathrm{a} *}$, Weiguang Yang ${ }^{1}$, Ziyao Zou ${ }^{1}$, Chen $\mathrm{Chen}^{1}$ and Xin Wang ${ }^{1}$ \\ ${ }^{1}$ College of Information and Control Engineering, Shenyang Jianzhu University, \\ Shenyang 110168; China \\ a455265987@qq.com \\ *The Corresponding author:
}

\section{Keywords: Smart home; Wireless sensor; Network routing protocol}

\begin{abstract}
The traditional smart home control and communicate household facilities by the wiring way of the cable lines generally, but this way of cable not only makes people struggle to break from the bondage of all kinds of cables and installation cost is higher, and the expansibility of the system is also very poor. The smart home based on wireless network technology can not only get rid of the bondage of cable and reduce installation cost, and scalability of the system is also a substantial improvement. So the research on the development of smart home wireless intelligent household is very necessary.
\end{abstract}

\section{Introduction}

Wireless sensor network (WSN) is an application of the network, the different application environment for wireless sensor network has different effects. The purpose of this paper lies in the application of intelligent household environment put forward an effective routing algorithm, ensure the effective information transmission, gathering together.

\section{Design of Wireless Sensor Network Technology}

Wireless Sensor Network is deployed in monitoring area from a large number of low-cost micro Sensor nodes, through wireless communication mode to form a multiple hops self-organizing Network, its purpose is collaboration to perceive, collection and processing network coverage area of perception object information, and sent to the observer. Sensors, object and perception observer constitute the three elements of the sensor network.

Each Sensor Node, Usually Has Data Acquisition, Data Processing And Data Transmission Function, Generally By The Sensor Module, Processing Module, Wireless Communication Module And Power Supply Module Four Parts. The Sensor Module Is Responsible For Monitoring Area Information And Data Transfer; Processor Module Is Responsible For The Control Of The Whole Node Operation, Store And Process Data Collected By Itself And The Data From The Other Node; Wireless Communication Module Is Responsible For The Wireless Communication With Other Sensor Nodes, Exchange Control Information And To Send And Receive Data; Power Supply Module For Sensor Nodes Provide The Energy Needed To Run.

Sensor Network Is Influenced By Many Aspects Of Practical Use, Including Error Tolerance, Scalability, Cost And Environment, Topology Control, Hardware Limitations And So On. These Factors Are Very Important, Because They Are The Study Of Wireless Sensor Network Protocols And Algorithms Have Very Important Influence.

(1) Tolerance.

Tolerate Sexual Means Under The Condition Of The Individual Node Failure Can Still Maintain The Original Function Of The Characteristics Of The Entire Network. Tolerance Should Be Considered In The Design Of Protocols And Algorithms.

\section{(2) Extensibility.}

The Design Of Wireless Sensor Network Should Be Able To Adapt To Different Sensor Distribution Density And The Number, At The Same Time For The Increase Of The Number Of Reduce Work Very Well. 
(3) Product Costs.

Reduce Costs Is To Wireless Sensors For Promotion And Application In Various Fields.

(4) Hardware Restrictions.

Each Node Includes Four Basic Parts: Monitoring, Calculation, To Send And Receive, Energy Control.

(5) Nodes of the Network Topology.

In General Topology Control Includes Several Stages: Early Layout Stage, Topology Maintenance Phase, Rearranging Stage, Etc.

(6) Environmental Factors.

The Sensor Network Is Geared To The Needs Of Specific Application Of Network, The Application Of Different, Have Different Characteristics.

(7) Transmission Medium.

The Wireless Sensor Network Generally Work At 433 MHZ, 915 MHZ, 2.4 Ghz.

There Is Also A Great Influence On Sensor Energy Factors, As Well As Data Transmission, Data Integration And So On.

\section{Wireless Sensor Networks and the Smart Home System}

The ultimate purpose of smart home system is to give the user more convenient and safe life, so a good smart home system should have low cost, simple to use, good flexibility, high stability, safety, etc. Smart home system master control chip and the selection of wireless sensor network in household network for smart home system cost, stability, security, flexibility and interoperability will have a major impact.

Control of Smart Home System and Selection of Wireless Sensor Network. The choice of smart home system master system control is the core of the smart home system. Currently on the market of smart home system control adopts ARM processor, and carry on ARM processor platform Linux or Android operating system, combined with the LCD display unit, which controls the intelligent household system for users to send commands. But the user wants to get a low cost, easy to use smart home system design scheme. If through a web control of household equipment, master in smart home system created for embedded Web server, the user does not need to install the client software, can pass any Internet equipment such as mobile phones, computers, open the web server page control of household equipment, at the same time, intelligent household system if lower demand for LCD display unit, also can to reduce the requirement of main control chip.

The Choice of Wireless Sensor Network in the Smart Home System. The construction of a wireless sensor network in household network is an important part of the smart home system. Living in network security and reliability of control commands transmission, rate is a major based on smart home wireless sensor network selection. According to the actual needs of smart home system, home network in smart home system mainly using wireless sensor network in household control command, on the basis of meet the demand of basic functions, also need to live in wireless sensor network node in the network with smaller size and strong flexibility. In conclusion, the intelligent household system for wireless sensor network node should have low cost, low rate, low power consumption, small volume, and the characteristics of high flexibility.

In traditional intelligent household system build home network use cable connection, more cable connection with high cost, complex wiring, difficult maintenance, beautiful affect indoor, etc. So the internal network will be used in smart home wireless network technology has become the trend of The Times, the following several kinds of commonly used short distance wireless communication technology, Bluetooth technology, Infrared technology, wi-fi Technology, Zig Bee technology and based on the Basic - RF two-way communication technologies.

The Remote Control of the Smart Home Technology. The stability of the remote control and convenience is one of the key elements of smart home system, at present commonly used by short messages, digital radio, CDMA, GPRS, in the form of Internet network and intelligent household transmission of the remote control command. At present domestic smartphone spread slowly, mobile application of portable computer also show explosive growth, if in the smart home system 
master control module based embedded Web server, the user can use any Internet devices (mobile phones, computers) to open the web page or use the PC client software, can complete the remote control of smart home system.

But considering if a user without the Internet, or not convenient to get to the Internet area, also need to remote control, smart home system now available to send short information remote control household equipment. And when a fire or gas leakage, users will receive alarm prompt message in a timely manner. In smart home system to make use of embedded web server, on the basis of the remote control, remote control mode combined with short information, will allow users to environment in any places conveniently completed the stability control of smart home system.

Requirements Analysis of Smart Home System Requirements. At present, the intelligent household system commonly used in high-grade hotels, residential area and mass approximately. The smart home system of ordinary people family didn't get a wide range of promotion. In addition to the current users of smart home recognition is still relatively low, there are three main reasons, first is the complex wiring, is not conducive to the construction of residential inside decorate installation; Second, the lack of smart home system can be customized by the user personalized services; Finally, the smart home system of the high cost of general intelligent household in the large-scale promotion of the people.

\section{Conclusion}

Connected with the wireless sensor network (WSN), these simple nodes can work under the state of a single intervention for a long time. By deploying this low power consumption, low rate of sensor nodes, people can be convenient for all kinds of information collection. The characteristics of its deployment are simple, convenient application makes people more and more inclined to the technology.

\section{Acknowledgments.}

Project supported by the programs of college students ' innovation and entrepre-neurship (No.201610153037; 201610153084; 2017X06097).

\section{Reference}

[1] PENG Junjie, He Hui, ZHU Pingan, LIU Yanping,ZHANG Xuejun, JIN Yi. Zigbee-based new approach to smart home[J]. Journal of Shanghai University (English Edition),2010,01:12-16.

[2] QIN Haichun,ZHAO Cong,WU Bo,ZHANG Li. Nursing bed used in the smart home environment[J]. Computer Aided Drafting,Design and Manufacturing, 2013,01:58-61.

[3] S.M.T.Bathaee, A.Fereidunian, A.Khajeh Amiri Hagh,H.Heydari. Design and Implementation of a Novel HomePlug-Based Solution for Low Cost and High Performance Smart Home Networking[J]. Journal of Electronic Science and Technology,2014,01:33-38.

[4] WANG Guang-wei,LU Sheng-li. Smart Home Gateway Based on ZigBee Technology[J]. International Journal of Plant Engineering and Management, 2015,04:240-249.

[5] Zou L,Chen L,Özsu M T.K-automorphism:a general framework for privacy preserving network publication[J]. Proceedings of the VLDB Endowment, 2009,2(1):946-957.

[6] Chen R,Philip S Y.Correlated network data publication via differential privacy[J].The International Journal on Very Large Data Bases,2014, 23(4): 653-676. 